\title{
Quantum-Critical Dynamics of the Skyrmion Lattice.
}

\author{
A. G. Green
}

October 30, 2018

\begin{abstract}
Near to filling fraction $\nu=1$, the quantum Hall ferromagnet contains multiple Skyrmion spin excitations. This multi-Skyrmion system has a tremendously rich quantum-critical structure. This is simplified when Skyrmions are pinned by disorder. We calculate the nuclear relaxation rate in this case and compare the result with experiment. We discus how such measurements may be used to further probe the quantum-critical structure of the multi-Skyrmion system.
\end{abstract}

At exact filling of a single Landau level the quantized Hall state forms an almost perfect ferromagnet. This quantum Hall ferromagnet (QHF) has some novel features due to the phenomenology of the underlying quantized Hall state. Magnetic vortices, or Skyrmions, in the QHF carry quantized electrical charge 1 . These Skyrmions are stabilised by a chemical potential so that the ground state slightly away from filling fraction $\nu=1$ contains a finite density of them. This original proposal of Sondhi et al. has been confirmed in a number of experiments [1, 2].

The $T=0$ phase diagram of the multi-Skyrmion system has been thoroughly investigated. In the absence of disorder, crystalline arrangements are

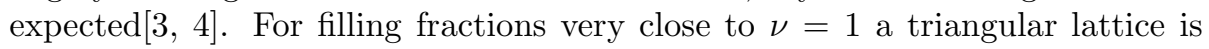
formed with a transition to a square lattice as the deviation from $\nu=1$ is increased. The statistical mechanics of the possible melting transitions has been considered [5]. At the highest Skyrmion densities, zero point fluctuations are expected to give rise to a quantum-melted state [6].

Despite this wealth of study, a complete account of the experimental observables has not been achieved. For example, nuclear magnetic resonance provides one of the clearest probes of the spin polarisation in the QHF[2]. Although it is understood in general terms how low-energy spin-fluctuations of the Skyrmion system may enhance the relaxation of nuclear spins, attempts to calculate relaxation rates have been flawed[7]. The fundamental physics, missed in other considerations, is the quantum-critical nature of fluctuations of the Skyrmion lattice. One immediate consequence of this quantum-criticallity is that the 
limits of temperature/frequency $\rightarrow 0$ and frequency/temperature $\rightarrow 0$ are very different. Typically, experimental probes are at frequencies much less than temperature and the latter limit is appropriate. This means that zero-temperature calculations cannot model experiments correctly [8].

Let us consider these points a little further. In our analysis below, we will find an underlying gappless XY-model governing orientational fluctuations of the multi-Skyrmion system. If a spinwave expansion is attempted for a gappless magnet at or below its critical dimension, the occupation of low-frequency modes is found to diverge. The constraint, fixing the magnitude of the local spin, restricts this divergence. Interplay between divergence and constraint gives rise to a finite (temperature dependent) correlation length, $\xi(T)$, beyond which correlations of the magnet decay exponentially. The dynamics of the critical magnet are very different on length scales greater or less than $\xi$. On lengthscales less than $\xi$, the groundstate is ordered (albeit in a quantum superposition of all possible orientations due to long wavelength spin fluctuations). Fluctuations with wavelength less than $\xi(T)$ may, therefore, be described by a modified spinwave expansion. On lengthscales greater than $\xi$, the groundstate is disordered and fluctuations are overdamped. This quantum relaxational dynamics is a striking feature of quantum-critical systems and leads to interesting universalities [9]. Regimes of renormalized classical and quantum activated behaviour at low-temperature, cross over to universal behaviour in the hightemperature quantum-critical regime.

The Skyrmion spin-configuration consists of a vortex-like arrangement of inplane components of spin with the z-component reversed in the centre of the Skyrmion and gradually increasing to match the ferromagnetic background at infinity. At large distances, the spin distribution decays exponentially to the ferromagnetic background on a length scale determined by the ratio of spin stiffness to Zeeman energy [10]. An individual Skyrmion may be characterized completely by its position (i.e. the point at which the spin points in the opposite direction to the ferromagnetic background) its size (i.e. the number of flipped spins) and the orientation of the in-plane components of spin. The equilibrium size of the Skyrmion is determined by a balance between its coulomb and Zeeman energies [1] (In the presence of a disorder potential, the potential energy of the Skyrmion also enters this balancing act 11]).

Consider a ferromagnet with a dilute distribution of Skyrmions. The normal modes of this system are relatively easy to identify. Firstly, ferromagnetic spinwaves propagate in-between the Skyrmions. The spectrum of these is gapped by the Zeeman energy and will be ignored from now on. Positional fluctuations, or phonon modes, of the Skyrmions are gapless in a pure system, but gapped when the lattice is pinned by disorder. Finally, fluctuations in the in-plane orientation and size must be considered. These two types of fluctuation are intimately connected; rotating a Skyrmion changes its size. This follows from the commutation relations of quantum angular momentum operators 4 , 7, , 12, 13].

The orientation, $\theta\left(\mathbf{x}_{i}, t\right)$, of a Skyrmion centred at a point $\mathbf{x}_{i}$ is described by 
the following effective action[7], 12]:

$$
S=\frac{1}{2} \int d t\left[\sum_{i} I_{i} \theta\left(\mathbf{x}_{i}, t\right) \partial_{t}^{2} \theta\left(\mathbf{x}_{i}, t\right)-\sum_{<i, j>} J_{i j} \cos \left(\theta\left(\mathbf{x}_{i}, t\right)-\theta\left(\mathbf{x}_{j}, t\right)\right)\right] .
$$

$I_{i}$ is the moment of inertia of the $i^{\text {th }}$ Skyrmion and $J_{i j}$ is the stiffness to relative rotations of neighbouring Skyrmions. The first term in Eq.(11) arises due to the change in energy of a Skyrmion when its size fluctuates; $\Delta E=I^{-1} \delta s^{2} / 8$. Since the z-component of spin and orientation are conjugate coordinates, a crossterm $i \delta s\left(\mathbf{x}_{i}, t\right) \partial_{t} \theta\left(\mathbf{x}_{i}, t\right) / 2$ appears in their joint effective action. Integrating out $\delta s\left(\mathbf{x}_{i}, t\right)$ gives the first term in Eq.(1). Clearly, 1/4I is the second derivative of the Skyrmion energy with respect to its spin. $I$ is related to the Skyrmion size (in fact $I=24 \mu_{B} g B / s$ 团) and, in the absence of disorder, is the same for all Skyrmions. Correlation functions involving $\delta s$ may be calculated using Eq.(11) by making the replacement $\delta s\left(\mathbf{x}_{i}, t\right) \rightarrow 2 i I \partial_{t} \theta(\mathbf{x}, t)$ - the result of a simple Gaussian integration over $\delta s$. The second term in Eq.(Ii) is an effective dipole-interaction of Skyrmions due to the energetics of overlapping Skyrmion tails [4, 13]. In a square Skyrmion lattice 14], $J_{i j}$ is independent of lattice site. A continuum limit may be taken where $\theta_{i}$ is replaced by a staggered field, $\theta_{i} \rightarrow \theta_{i}+\eta_{i} \pi$, with $\eta_{i}=0,1$ on adjacent sites, and $\theta_{i}-\theta_{j}$ is replaced by a derivative;

$$
S=\frac{1}{2} \int \frac{d \omega d^{2} k}{(2 \pi)^{3}} \theta(\mathbf{k}, \omega)\left(\delta \nu \bar{\rho} I \omega^{2}+J \mathbf{k}^{2}\right) \theta(-\mathbf{k},-\omega) .
$$

The frequency integral in this expression is shorthand for a Matsubara summation at finite temperature and the momentum integral is over the Brillouin zone 15. A factor of the Skyrmion density $\delta \nu \bar{\rho}$ has been introduced, where $\delta \nu$ is the deviation from filling fraction $\nu=1$ and $\bar{\rho}$ is the electron density. There are a few caveats to the use of Eq.(2). We defer discusion of these until later. This model is perhaps most familiar as an effective theory of the Josephson junction array. In this case, $\theta$ is the phase of the superconducting order parameter and its conjugate coordinate is the charge of the superconducting junction.

In order to calculate properties of the Skyrmion lattice, we must relate fluctuations in the Skyrmion orientation to fluctuations in the orientation of local spin. We use a coherent-state representation of the polarization of the local spin, via an $\mathrm{O}(3)$-vector field $\mathbf{n}(\mathbf{x}, t)$. The static spin distribution at a point $\mathbf{x}$ relative to the centre of a single Skyrmion is denoted by $\mathbf{n}(\mathbf{x})$ and its in-plane components by $n_{x}+i n_{y}=n_{r} e^{i \phi_{0}}$. The in-plane components of local spin at a point $\mathbf{x}$, in response to rotational fluctuations of a Skyrmion centred at $\mathbf{x}_{i}$, are given by

$$
n_{r} e^{i \theta}(\mathbf{x}, t)=(-)^{\eta_{i}}\left[n_{r}\left(\mathbf{x}-\mathbf{x}_{i}\right)+2 i I \frac{\partial n_{r}\left(\mathbf{x}-\mathbf{x}_{i}\right)}{\partial s} \partial_{t} \theta\left(\mathbf{x}_{i}, t\right)\right] e^{i \theta+i \phi_{0}}\left(\mathbf{x}_{i}, t\right) .
$$

We have used the conjugate relationship between Skyrmion spin and orientation in writing down this expression. In a distribution of many Skyrmions, one must 
in principle sum the contributions of all Skyrmions to the fluctuation in spin at the point $\mathbf{x}$. However, in the dilute limit in which we are performing our explicit calculation, Skyrmions are exponentially localized. The dominant spin fluctuations occur near to the centre of Skyrmions and so, to logarithmic accuracy, the local fluctuations at a point $\mathbf{x}$ are due only to the nearest Skyrmion.

We will calculate the nuclear relaxation rate due to low energy quantum fluctuations of the Skyrmion lattice;

$$
\frac{1}{T_{1}}=T \gamma \lim _{\omega \rightarrow 0} \int \frac{d^{2} k}{(2 \pi)^{2}} \frac{\mathcal{I} m\left\langle S_{+}(\mathbf{k}, \omega) S_{-}(-\mathbf{k},-\omega)\right\rangle}{\omega},
$$

where $\gamma$ is the hyperfine coupling constant. Other physical observables, such as the temperature dependence of magnetization, $\langle M\rangle=\int d t d^{2} x\left\langle n_{z}(\mathbf{x}, t)\right\rangle$, may be calculated similarly. Our first task is to replace the expectation of the spin raising and lowering operators in Eq.(任) by correlators of the Skyrmion orientation. Substituting from Eq.(3) into Eq. (4) and ignoring terms higher order in frequency, the nuclear relaxation rate at a point $\mathbf{x}$ is

$$
\frac{1}{T_{1}}(\mathbf{x})=T \gamma \lim _{\omega \rightarrow 0} n_{r}^{2}(\mathbf{x}) \int \frac{d^{2} k}{(2 \pi)^{2}} \frac{\mathcal{I} m\left\langle e^{i \theta}(\mathbf{k}, \omega) e^{-i \theta}(-\mathbf{k},-\omega)\right\rangle}{\omega} .
$$

This takes the form of a correlation function of the Skyrmion orientation, multiplied by a profile function characteristic of the Skyrmion groundstate. The average rate is given by integrating this over an area containing a single Skyrmion and multiplying by the Skyrmion density $\delta \nu \bar{\rho}$. The result is identical to Eq.(5) with the replacement $n_{r}^{2}(\mathbf{x}) \rightarrow \delta \nu \overline{n_{r}^{2}}$. $\overline{n_{r}^{2}}=\bar{\rho} \int d^{2} x n_{r}^{2}(\mathbf{x})$ is a number characteristic of a single Skyrmion. For a pure Skyrmion spin distribution [10], $\overline{n_{r}^{2}}=2 s$ to logarithmic accuracy in the Skyrmion spin, $s=\bar{\rho} \int d^{2} x\left(1-n_{z}(\mathbf{x})\right)$. Notice that radial fluctuations of local spin contribute only to higher order in frequency and have been neglected in writing down Eq.(5).

The problem of finding the nuclear relaxation rate has now been reduced to evaluating the correlation function in Eq.(5) using the effective action Eq.(2). This is rather tricky. The $\mathrm{O}(2)$-quantum rotor, described by Eq.(2), is quantumcritical. It is necessary to employ a non-perturbative scheme, such as $1 / \mathrm{N}$ or epsilon expansions, to calculate in the quantum-critical regime of this model. Here we use the result of the $1 / \mathrm{N}$ expansion of Chubukov et al 16 .

An important feature of the effective action, Eq.(2), is that it displays a zero-temperature phase transition. For $I J<1$, the Skyrmion moment of inertia is sufficiently small that quantum fluctuations destroy long range order even at zero temperatures. For $I J>1$, the $T=0$ groundstate has an infinite correlation length and is ordered. Notice that arbitrarily small temperatures destroy this long-range order even when $I J>1$. This arises due to the interplay between fluctuations and constraint and can be seen in a simple mean-field calculation 16. In the $\mathrm{O}(2)$ representation of Eq.(2), $S=$ $\int d t d^{2} x\left[\mathbf{n}\left(I \partial_{t}^{2}+J \partial_{\mathbf{x}}^{2}\right) \mathbf{n}+\lambda(\mathbf{x}, t)\left(\mathbf{n}^{2}-1\right)\right]$, where $\mathbf{n}(\mathbf{x}, t)$ is an $\mathrm{O}(2)$-vector field and $\lambda(\mathbf{x}, t)$ is an auxiliary field that imposes the constraint $\mathbf{n}^{2}=1$. Imposing the constraint at mean-field level, $\left\langle\mathbf{n}^{2}\right\rangle=1$, determines a temperature 


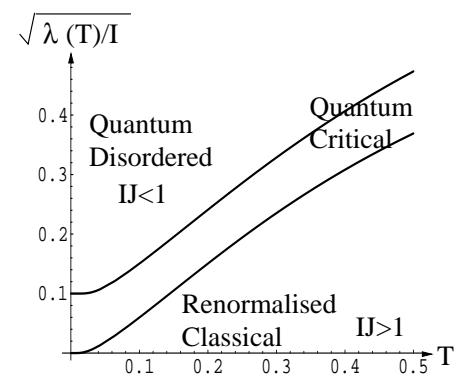

Figure 1: T-dependence of the Gap.

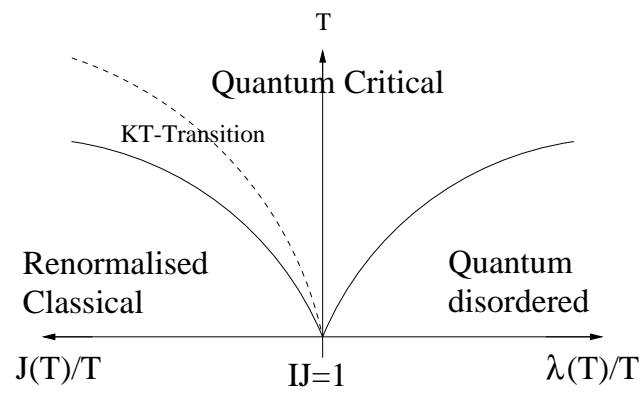

Figure 2: Phase diagram of the $\mathrm{O}(2)$-vector model.

dependent gap, $\lambda(T)$. The spin correlations decay exponentially on a length scale $\xi(T)=\sqrt{J / \lambda(T)}$. The results of such a calculation are sketched in Fig.1. Above a temperature of about $T_{Q C}=\left|2 \pi J-E_{\max }\right|$ (The cut-off, $E_{\max }=2 \pi \sqrt{J / I}$ corresponds to fluctuations with momentum at the Brillouin zone boundary, $k_{\max }=\pi \sqrt{\bar{\rho} \delta \nu}$ 15]), the gap/correlation length develops a universal temperature dependence. In this region, thermal and quantum fluctuations are of similar importance and are very difficult to disentangle. This crossover to universal high-temperature behaviour from distinct lowtemperature behaviours is a feature of all correlation functions of Eq. (2). It is usual to summarise this behaviour by the phase diagram sketched in Fig.2. In this figure, $J(T)$ is the renormalized stiffness in the ordered phase and $\lambda(T)$ is the gap in the paramagnetic phase.

The correlation function required in Eq.(5) has been calculated in Ref.[16] by means of a $1 / \mathrm{N}$ expansion, with the result

$$
\begin{array}{rlrl} 
& \lim _{\omega \rightarrow 0} \frac{1}{\omega} \int \frac{d^{2} k}{(2 \pi)^{2}} \mathcal{I} m\left\langle e^{i \theta}(\mathbf{k}, \omega) e^{-i \theta}(-\mathbf{k},-\omega)\right\rangle \\
= & \frac{1}{T} \frac{0.015}{\sqrt{I J}}\left(\frac{k T}{\pi J}\right)^{\eta}, & T \gg T_{Q C} \\
= & \frac{1}{T} \frac{0.085}{\sqrt{I J}} \frac{I T^{2}}{\lambda(0)} e^{-2 \sqrt{\lambda(0) / I T^{2}},} & T \ll T_{Q C}, & I J<4, \\
= & \frac{0.18}{T}\left(\frac{k T}{2 \pi \lambda(T)}\right)^{1 / 2}, & T \ll T_{Q C}, & I J>4,
\end{array}
$$




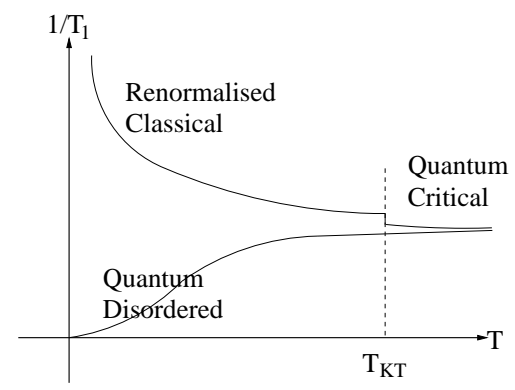

Figure 3: Temperature of the nuclear relaxation rate, $1 / T_{1}$.

where $\eta$ is a number close to zero. Substituting these results into Eq.(5) we obtain

$$
\frac{1}{T_{1}}=\gamma \frac{0.03 s}{\sqrt{I J}}\left(\frac{k T}{\pi J}\right)^{\eta}
$$

at high temperature. The full behaviour is sketched in Fig.3. The kink at $T_{K T}$ is due to the discontinuous change in spin stiffness seen at the Kosterlitz-Thouless transition. This effect is not seen in the $1 / \mathrm{N}$ expansion of Ref.[16] and must be calculated by some other means.

The nuclear relaxation rate obtained here is very different from that obtained in Ref. [7]. As pointed out in the introduction, this is due to the unphysical limit $T / \omega \rightarrow 0$ used in Ref.[7]. Nevertheless, it is instructive to see how the results of Ref.[7] relate to the present formalism. Since $T \ll \omega$ in their work, Côté et al consider fluctuations on length scales much less than the correlation length. The groundstate is ordered and a spinwave expansion may be used. Long wavelength fluctuations lead to a superposition of orientations- rotational averagingthe immediate consequence of which is that $\left\langle e^{i \theta}(\mathbf{k}, \omega) e^{-i \theta}(-\mathbf{k},-\omega)\right\rangle=0$. Returning to the substitution of Eq.(3) into Eq.(勾), we must retain terms to next order in frequency. This is a cross-term between radial and transverse fluctuations and involves a correlator $\left\langle\partial_{t} \theta e^{i \theta}(\mathbf{k}, \omega) e^{-i \theta}(-\mathbf{k},-\omega)\right\rangle$. Evaluating this correlation function via a $T=0$ spinwave expansion of the effective action, Eq.(2), reproduces the result of Ref.[7] (up to a numerical factor due to our estimate of the Skyrmion profile function, $n_{r} \partial n_{r} / \partial s$ ). The zero-temperature phonon contribution may be calculated similarly.

Up to now, we have assumed that the Skyrmion lattice is pinned by disorder and that phonons may be ignored as a consequence. The situation is rather subtle and a fuller discussion is appropriate at this juncture. A quadratic effective action for phonons of the Skyrmion lattice is known 3, 4, 5]. It is identical to that of an electronic Wigner crystal in a magnetic field with a vanishing effective mass 17. Under this effective action, Skyrmions move in small ellipses with a frequency $\omega_{\mathbf{k}} \sim|\mathbf{k}|^{3 / 2} / B$ and major axes orientated transverse to the phonon momentum.

At finite temperature, the occupation of transverse phonons is infra-red divergent. This divergence is restricted by interactions between phonons arising 
from the non-harmonicity of the Skyrmion interaction. Unlike fluctuations in orientation, where the spinwave interaction is due to a topological constraint, these phonon interactions are non-universal (at best the universality is hidden in the details of the groundstate spin distribution and effective Skyrmion interaction potential). The resulting physics is very similar to that discussed for the rotation mode above; the phonon system is quantum-critical and has lowtemperature ordered and quantum-melted [6] phases and a high-temperature quantum-critical regime.

Even this is not the full story. Although the low energy dispersions of phonons and Skyrmion rotations are independent [7], non-linear interactions exist between these modes. The lattice stiffness is, in part, due to the dipole interaction of Skyrmions and is affected by fluctuations in orientation. Similarly, the dipole interaction between Skyrmions is strongly dependent upon the separation of Skyrmions and is affected by phonons. These non-linearities occur on the same footing as the phonon-phonon interactions and rotation-rotation interactions. The full quantum-critical structure of the multi-Skyrmion system is tremendously complicated.

The position taken here in neglecting this wealth of structure is that the Skyrmion lattice is pinned by disorder and the phonon spectrum gapped. Phononic fluctuations are suppressed at low temperatures and the associated critical structure occurs at higher temperature. The residual effect of phonons is a slight thermal renormalization of the rotational stiffness. The slight distortion in static positions of Skyrmions, in response to the disorder potential, gives a small random contribution to the stiffness $J$. This randomness produces a small region of Bose-glass phase at low temperatures, intervening between the paramagnet and renormalized classical regimes. For weak disorder, this phase only affects the physics very close to the critical point and does not affect our conclusions.

We now turn to a discussion of the experimental implication of the above calculations. Detailed measurements of $1 / T_{1}$ have been carried out by Bayot et al 18]. Above $40 \mathrm{mK}, T_{1}$ is independent of temperature. This is consistent with the rotational degrees of freedom being in their quantum-critical regime. Values of $I$ and $J$ for this system extracted from TDHFA calculations close to 1 . The system is close to criticality and the crossover to the quantumcritical regime occurs at correspondingly low temperature. At $40 \mathrm{mK}$ there is an abrupt step in $1 / T_{1}$ (and attendant peak in heat capacity). This is consistent with a Kosterlitz-Thouless transition in the orientational order (notice that the crossover temperature $\left|2 \pi J-E_{\max }\right|$ may be much less than $T_{K T}=2 \pi J$ and so the behaviour may be quantum-critical either side of the transisiton). There are a number of other candidate transitions 3 , 5, 7], however, and it is not easy to discriminate between them. Considerations along the lines of those presented here allow some elaboration, but this is necessarily rather speculative and we refrain from its discussion at present. We may make some firm predictions for nuclear relaxation measurements below $40 \mathrm{mK}$. Changing the deviation in filling fraction or using tilted filed measurements both change the parameter $I J$ and allow exploration of the phase diagram shown in Fig.1. The divergence or otherwise of the nuclear relaxation rate as temperature is reduced to zero should 
give a clear indication of the quantum-critical structure.

I would like to thank N. R. Cooper, J. R. Chalker, S. M. Girvin and N. Read for enlightening discusions, comments and suggestions. This work was supported by Trinity College Cambridge.

\section{References}

[1] S. L. Sondhi, A. Karlhede, S. A. Kivelson and E. H. Rezayi, Phys. Rev. B47, 16419 (1993).

[2] S. E. Barrett, G. Dabbagh, L. N. Pfeiffer, K. W. West and R. Tycko, Phys. Rev. Lett. 745112 (1995); R. Tycko, S. E. Barrett, G. Dabbagh, L. N. Pfeiffer and K. W. West, Science 268, 1460 (1995); E. H. Aifer, B. B. Goldberg, D. A. Broido, Phys. Rev. Lett. 76680 (1996); A. Schmeller, J. P. Eisenstein, L. N. Pfeiffer and K. W. West, Phys. Rev. Lett. 75, 4290 (1995).

[3] L. Brey, H. A. Fertig, R. Côté and A. H. MacDonald, Phys. Rev. Lett. 76, 2562 (1995); A. G. Green, I. I. Kogan and A. M. Tsvelik, Phys. Rev. B 54 16838 (1996); M. Rao, S. Sengupta and R. Shankar, Phys. Rev. Lett. 79, 3998, (1997);

[4] M. Abolfath and M. R. Ejtehadi, cond-mat/9807236 (1998); Phys. Rev. B58, 10665 (1998).

[5] Carsten Timm, S. M. Girvin, H. A. Fertig, Phys. Rev. B58, 10634 (1998).

[6] B. Paredes and J. J. Palacios, cond-mat/9902031 (1999).

[7] R. Côté, A. H. MacDonald, Luis Brey, H. A. Fertig, S. M. Girvin and H. T. C. Stoof, Phys. Rev. Lett. 78, 4825 (1997); cond-mat/9702207 (1997).

[8] Similar considerations are key in the calculation of finite temperature conductivity of the 2-dimensional supercondutor. K. Damle and S. Sachdev, Phys. Rev. B 568714 (1997).

[9] N. Read and S. Sachdev, Phys. Rev. Lett. 75, 3509 (1995).

[10] The pure Skyrmion spin distribution has the form $\left(n_{x}+i n_{y}\right)(\mathbf{r})=2(x+$ iy) $e^{i \theta} r_{0} /\left(r^{2}+r_{0}^{2}\right), n_{z}(\mathbf{x})=\left(r^{2}-r_{0}^{2}\right) /\left(r^{2}+r_{0}^{2}\right)$, out to a radius $\sqrt{\rho_{s} / \bar{\rho} g B}$, beyond which the spin distribution decays exponentially to the ferromagnetic groundstate. $r_{0}$ is a parameter related to the spin of the Skyrmion and $\theta$ its orientation.

[11] A. J. Nederveen and Yuli. V. Nazarov, Phys. Rev. Lett. 82, 406 (1999).

[12] Yuli V. Nazarov and A. V. Khaetski, Phys. Rev. Lett. 80, 576 (1998).

[13] B. M. A. G. Piette, B. J. Schroers and W. J. Zakrzewshi, Nucl. Phys. B 439, 205 (1995). 
[14] In the case of the hexagonal lattice, anti-ferromagnetic order is frustrated and a different description is required.

[15] The Skyrmion lattice has an anti-ferromagnetic ordering of in-plane spins. The unit cell, therefore, contains two Skyrmions and the Brillouin zone must be chosen appropriately.

[16] A. V. Chubukov, S. Sachdev and J. Ye, Phys. Rev. B49, 11919 (1994).

[17] A curious feature of the nearly filled Landau level is that quasi-particles have only a magnus-force term in their effective action and no kinetic term.

[18] V. Bayot, E. Grivei, S. Melinte, M. B. Santos and M. Shayegan, Phys. Rev. Lett. 76, 4585 (1996); V. Bayot, E. Grivei, J.-M. Beuken, S. Melinte and M. Shayegan, Phys. Rev. Lett. 79, 1718 (1997). 